DR. YUSUF A. RAJABALLY (Orcid ID : 0000-0002-7170-8343)

Article type : Editorials

\title{
Compliance with international guidelines for chronic
}

\section{inflammatory neuropathies}

Yusuf A. Rajabally, ${ }^{1} \quad$ Peter van den Bergh, ${ }^{2}$ for the European Study Group on

Guidelines for Neuropathy*

1. School of Life \& Health Sciences, Aston Brain Centre, Aston University, Birmingham, $U . K$.

2. Centre Neuromusculaire, Cliniques Universitaires St. Luc, Université Catholique de Louvain, Brussels, Belgium.

*Adrian Fowle, ${ }^{3}$ Jean-Marc Leger, ${ }^{4}$ Eduardo Nobile-Orazio, ${ }^{5}$ Antonino Uncini ${ }^{6}$ and Pieter van Doorn. ${ }^{7}$

3. Department of Clinical Neurophysiology, St. Peter's Hospital, Chertsey, U.K.

4. National Referral Center for Rare Neuromuscular Diseases, Hospital PitiéSalpêtrière, University Pierre et Marie Curie (Paris VI), Paris, France.

This article has been accepted for publication and undergone full peer review but has not been through the copyediting, typesetting, pagination and proofreading process, which may lead to differences between this version and the Version of Record. Please cite this article as doi: $10.1111 /$ ene. 13873

This article is protected by copyright. All rights reserved. 
5. Neuromuscular and Neuroimmunology Service, University of Milan, Milan, Italy.

6. Department of Neuroscience, Imaging and Clinical Sciences, University "G. d'Annunzio", Chieti, Italy.

7. Erasmus MC University Medical Centre, Rotterdam, Netherlands.

\section{Conflicts of Interest: None for this work}

Abbreviations: CIDP: chronic inflammatory demyelinating polyneuropathy; EFNS/PNS: European Federation of Neurological Societies/Peripheral Nerve Society; MMN: multifocal motor neuropathy

Correspondence to: Yusuf A. Rajabally, School of Life \& Health Sciences, Aston Brain Centre, Aston University, Aston Triangle, Birmingham B4 7ET, U.K. e-mail: y.rajabally@aston.ac.uk

ERare diseases' management guidelines are produced with the primary aim of improving practice and standards of care for patients and may represent a useful framework for clinical practice. The EFNS/PNS (European Federation of Neurological Societies/Peripheral Nerve Society) guidelines for CIDP (chronic inflammatory demyelinating polyneuropathy) and MMN (multifocal motor neuropathy) were last published in 2010 (1, 2). Enthusiasm of the audience for whom they are produced, arguably primarily non-sub-specialists, is however 
largely unexplored. Compliance to these guidelines by neuromuscular and/or peripheral nerve specialists has not been investigated.

In this issue of the journal, Rosier et al. describe the findings of their study specifically in relation to intravenous immunoglobulin (IVIg) treatment for these disorders (3). They aimed, through a survey, to evaluate French daily neurological practice in relation to the abovementioned published guidelines. They performed a retrospective observational study based on a pilot questionnaire validated by expert neurologists in the field. Four hundred and eleven neurologists were invited by e-mail to fill a general questionnaire and 4 patient case reports concerning recently seen or soon to be seen subjects. Of the 70 responders, 49 met predefined criteria for inclusion in the analysis. A minority (26.6\%) worked in neuromuscular reference centres (NRCs), coming from most (10 of the 13 existing) of the French NRCs, thereby offering an adequate global view of subspecialist practice. The authors mainly ascertained that initial treatment procedures for both CIDP and MMN conformed to existing guidelines. However, in greater detail, this essentially concerns the dose of $2 \mathrm{~g} / \mathrm{kg}$ used over 5 days, the 4-weekly administration frequency with dose maintenance for the first 3 courses. In contrast, in relation to longer term therapy and guideline recommendations, some major and interesting discrepancies were observed, notably with non-NRC neurologist decreasing significantly more frequently the dose of IVIg than NRC neurologists $(83 \%$ vs. $51 \%$; $p<0.05)$ and using immunosuppressants significantly less frequently $(39 \%$ vs. $92 \%$; $p<0.05)$. Immunosuppressants were used for nearly $40 \%$ of cases by neurologists and although the details are not provided, it would be likely this practice was more common in NRCs. Dose reductions are recommended in the guidelines and implementation of this, appears from the reported findings of Rosier et al., less widespread amongst subspecialists. One may argue that NRCs see more severely affected subjects, although how this may justify sustained high dose therapy, presumably in circumstances of stability or progressive and possibly treatment- 
unrelated improvement, for over a quarter of subspecialists for the first 12 treatment courses, is somewhat mystifying. This is compounded by the two-fold and four-fold higher follow-up frequencies of CIDP and MMN patients respectively, in NRCs compared to the general neurology setting, which one would have expected making dose reduction decisions easier. On the other hand, the use of immunosuppressants, for which there is no current evidence base, is not recommended by the existing guidelines neither for CIDP nor for MMN. Despite this lack of justification, subspecialists appear to widely diverge from the recommendations in their practice, as opposed to generalists. Also, of note, and in relation to published data on the usually fixed frequency requirements particularly in $\operatorname{CIDP}(4,5)$, all neurologists in contrast, more frequently altered treatment interval rather than dose (97\% vs. 65\% for CIDP and $98 \%$ vs. $51 \%$ for $\mathrm{MMN})$.

These findings in themselves clearly therefore suggest poor longer-term compliance to the guidelines, which importantly, but also perplexingly, appears to predominate in NRCs. That nearly a third of patients with CIDP and nearly half of those with MMN were referred by general neurologists to NRCs suggests that cases managed by subspecialists do not majorly differ in presentation or progression to those whose treatment remained in the hands of general neurologists. It is clear however that subsequent patient management differs significantly within as compared to outside NRCs, this in itself, irrespective of hypothetical underlying reasons, being inconsistent with the aim and objectives of guideline generation and dissemination.

Our group conducted an international web-based audit on compliance to EFNS/PNS Guidelines on CIDP and MMN between 2012 and 2015 in 9 countries in total: Italy, Belgium, Serbia, Moldova, France, Hungary, Portugal, the United Kingdom and the state of Kerala in India. The main finding of our audit was the low response rate (globally $4.2 \%$ of all contacted potential participants). Nearly $60 \%$ of non-neuromuscular neurologists stated that 
they used EFNS/PNS Guidelines to establish their therapeutic decision-making for CIDP and MMN. Nearly a sixth had however no knowledge of the Guidelines, the electrophysiological features being as expected, less commonly known than the clinical aspects. A large majority of neuromuscular neurologists preferred using EFNS/PNS criteria in clinical practice than any other criteria. Intravenous immunoglobulin dose reduction trials were, in contrast to Rosier et al.'s findings, reported as common practice for the majority of neuromuscular neurologists, for long-term patients. Although in lower proportions as compared to the French study, over $50 \%$ of neuromuscular neurologists declared feeling immunosuppression may be justified in CIDP, irrespective of the response to steroids, immunoglobulins or plasma exchange.

Our analysis, through the poor responder rate from a large, similarly sampled, e-mailed invited, international neurological cohort, demonstrates a low level of interest in guidelines for rare diseases such as CIDP and MMN, particularly amongst general neurologists. Furthermore, a selection bias is likely to have occurred, as illustrated by the contrasting high level of guideline awareness in the surveyed sample. A similar selection bias is also likely in Rosier et al.'s study. Our results indicate that the surveyed sample was aware of the guidelines, but similarly to the French cohort, behaved variably in relation to the recommendations within them.

Current EFNS/PNS guidelines represent very useful research tools as we have shown in a previous analysis (6). Awareness and compliance to these guidelines appear however, both from the data from Rosier et al. and from our international analysis, much more doubtful. In the French study, the poor long-term compliance amongst NRC neurologists enhances the concerns, indicating the need for greater coherence in the subspecialist arena, so as to improve newer versions and updates for all practitioners at large. New guidelines will be necessarily and rightly focused on the evidence base $(7,8)$. However, consideration of factors 
impacting on dissemination and uptake will equally require careful consideration. It may be hoped this will ultimately allow the improvement and harmonisation of standards of care of all affected patients.

\section{References}

1. European Federation of Neurological Societies/Peripheral Nerve Society guideline on management of multifocal motor neuropathy. Report of a joint task force of the European Federation of Neurological Societies and the Peripheral Nerve Society--first revision. Journal of the peripheral nervous system : JPNS. 2010;15(4):295-301.

2. Van den Bergh PY, Hadden RD, Bouche P, Cornblath DR, Hahn A, Illa I, et al. European Federation of Neurological Societies/Peripheral Nerve Society guideline on management of chronic inflammatory demyelinating polyradiculoneuropathy: report of a joint task force of the European Federation of Neurological Societies and the Peripheral Nerve Society - first revision. European journal of neurology. 2010;17(3):356-63.

3. Rosier C, Graveline N, Lacour A, Antoine JC, Camdessanche JP. IGIV for chronic inflammatory demyelinating polyneuropathies and multifocal motor neuropathies treatment in France: are daily practices in accordance with guidelines? European journal of neurology. 2018.

4. Lunn MP, Ellis L, Hadden RD, Rajabally YA, Winer JB, Reilly MM. A proposed dosing algorithm for the individualized dosing of human immunoglobulin in chronic inflammatory neuropathies. Journal of the peripheral nervous system : JPNS. 2016;21(1):337. 
5. Rajabally YA, Seow H, Wilson P. Dose of intravenous immunoglobulins in chronic inflammatory demyelinating polyneuropathy. Journal of the peripheral nervous system : JPNS. 2006;11(4):325-9.

6. Rajabally YA, Fowle AJ, Van den Bergh PY. Which criteria for research in chronic inflammatory demyelinating polyradiculoneuropathy? an analysis of current practice. Muscle \& nerve. 2015;51(6):932-3.

7. Guyatt GH, Oxman AD, Schunemann HJ, Tugwell P, Knottnerus A. GRADE guidelines: a new series of articles in the Journal of Clinical Epidemiology. Journal of clinical epidemiology. 2011;64(4):380-2.

8. Malmivaara A. Methodological considerations of the GRADE method. Annals of medicine. 2015;47(1):1-5.

This article is protected by copyright. All rights reserved. 